\title{
Improved production of sublancin via introduction of three characteristic promoters into operon clusters responsible for this novel distinct glycopeptide biosynthesis
}

Shengyue $\mathrm{Ji}^{1+}$, Weili $\mathrm{Li}^{1+}$, Abdul Rasheed Baloch${ }^{2}$, Meng Wang ${ }^{2}$ and Binyun Cao ${ }^{1 *}$

\begin{abstract}
Background: Sublancin is a novel and distinct antimicrobial glycopeptide that can be used as an alternative to conventional antibiotics. The reported production of sublancin by Bacillus subtilis 168 is poor because transcriptional regulatory circuit of sunA, a gene that encodes presublancin, is complex and difficult to control.

Results: A strong inducible and easy to control vegetative $\sigma^{A}$ promoter of $\mathrm{P}_{g / v}$ was introduced to replace that of sunA in situ in B. subtilis 1A747 [SP $\beta c$, prototroph, the derivative of B. subtilis 168 (trpC2)]. Meanwhile, other two strong promoters of P43 and $P_{l u x s}$ were respectively placed before sunl and sunT-bdbA-sunS-bdbB, encoding five functional proteins that involved in the biosynthesis of mature sublancin. $642 \mathrm{mg}$ sublancin was obtained from $1 \mathrm{~L}$ culture supernatant of recombinant B. subtilis $1 \mathrm{~A} 747$ strains. Analysises of electrospray ionization mass spectrometry and circular dichroism spectrum showed that mature sublancin had a molecular weight of 3877.642 Da and displayed a a-helical conformation that are consistent with reported results. In addition, the mature sublancin was proved to be a potent antimicrobial glycopeptide with broad activity spectrum, moderate cytotoxicity and good conditional stability under high temperature, extreme $\mathrm{pH}$ and protease-rich environments, thus showing its potential for clinical applications.

Conclusions: Our present findings suggest that recombinant B. subtilis 1 A747 strains can effectively and efficiently biosynthesize mature sublancin. The replacement of native promoters provides an extra method for production improvement of some other complicated peptides such as nisin and subtilin.
\end{abstract}

Keywords: Sublancin, Glycopeptide, Recombinant B. subtilis 1A747, Improved production, Transcriptional regulatory circuit

\section{Background}

Natural peptides with post-translational modification are rapidly expanding class of agents with diverse biological activities [1]. Sublancin (Genbank accession number P68577.1) is a novel distinct peptide that is synthesized by Bacillus subtilis 168. This peptide can effectively kill specific pathogenic bacteria such as Staphylococcus aureus and Streptococcus pyogenes [2]. Sublancin is encoded by SP $\beta$ prophage in strains that lysogenize the SP $\beta$ bacteriophage

\footnotetext{
*Correspondence: jiziqing2010@163.com

${ }^{\dagger}$ Equal contributors

'College of Animal Science and Technology, Northwest A\&F University, 22 Xinong Road, Yangling, 712100 Shaanxi, P.R. China

Full list of author information is available at the end of the article
}

and inhibits the growth of non-lysogenic strains [3]. Similar to lantibiotics [4], sublancin is firstly synthesized as a precursor with a double-glycine leader peptide in $\mathrm{N}$-terminal and a core peptide in C-terminal, and the latter was posttranslationally modified into mature peptide. However, unlike lantibiotics, sublancin has a unique post-translational S-glucosylation modification and is therefore considered as a distinct glycopeptide [5].

The DNA fragment responsible for biosynthesizing mature sublancin is located in the prophage SP $\beta$ genome and includes two adjacent transcriptional units (sunI and $\operatorname{sun} A-\operatorname{sun} T-b d b A-\operatorname{sun} S-b d b B$ ) with a length of $4.5 \mathrm{~kb}$ (Figure 1a). sunI provides the genetic basis for sublancin 
producer immunity [6]. Adjacently, an operon with five successive genes, $\operatorname{sun} A-\operatorname{sun} T-b d b A-s u n S-b d b B$, is located immediately downstream of sunI. However, a terminator structure is present between $\operatorname{sunA}$ and $\operatorname{sun} T$ that, in some events such as sporulation happens in the DSM media, causes premature termination of the transcript (Figure 1a and e). The whole transcript of sunAsun $T-b d b A-s u n S-b d b B$ is transcribed when mature sublancin is biosynthesized $[7,8]$. $\operatorname{sun} A$ is immediately located downstream of sunI and encodes the presublancin [2], and SunT is an ABC-type transporter with a proteolytic domain that removes the leader peptide from sublancin during its translocation across the membrane $[9,10]$. BdbA still remains unclear in sublancin biosynthesis although it has been presumed to have thiol oxidase activity [11]. BdbB belongs to the thiol-disulfide oxidoreductases and involved in the post-translational modification of disulfide bond formation in sublancin $[9,11,12]$. SunS is a S-glycosyltransferase that has a CxxS motif; and is involved in biosynthesis of mature sublancin by glucosylating $\mathrm{Cys}^{22}$
[13]. S-glycoside moiety of sublancin is important for conferring the antimicrobial activity [5].

Sublancin biosynthesis is controlled by a complex regulatory network that involves a minimum of five transcriptional regulators, namely Abh, AbrB, Rok, YvrG and YvrH (Additional file 1) $[7,14,15]$. Abh and AbrB directly bind to the overlapping regions within $\operatorname{sun} A$ regulatory region (Figure 1b). Abh plays a positive role in regulating the transition-stage $\operatorname{sun} A$ expression during vegetative growth. AbrB is a paralog of Abh that can transcriptionally repress the biosynthesis of sublancin [14,16]. The in vitro studies have shown that Rok can bind to sunA transcriptional regulatory region and its deletion improves the transcription of sunA and sunT [15]. YvrG and YvrH comprise of a novel two-component system, and simultaneously positively regulate the transcriptional units of sunA and sunT-bdbAsunS-bdbB [7].

ECF $\sigma$ factors belong to a subfamily of sigma 70 class and respond to various extracellular changes [17], and the regulation of antibiotic resistance functions is commonly
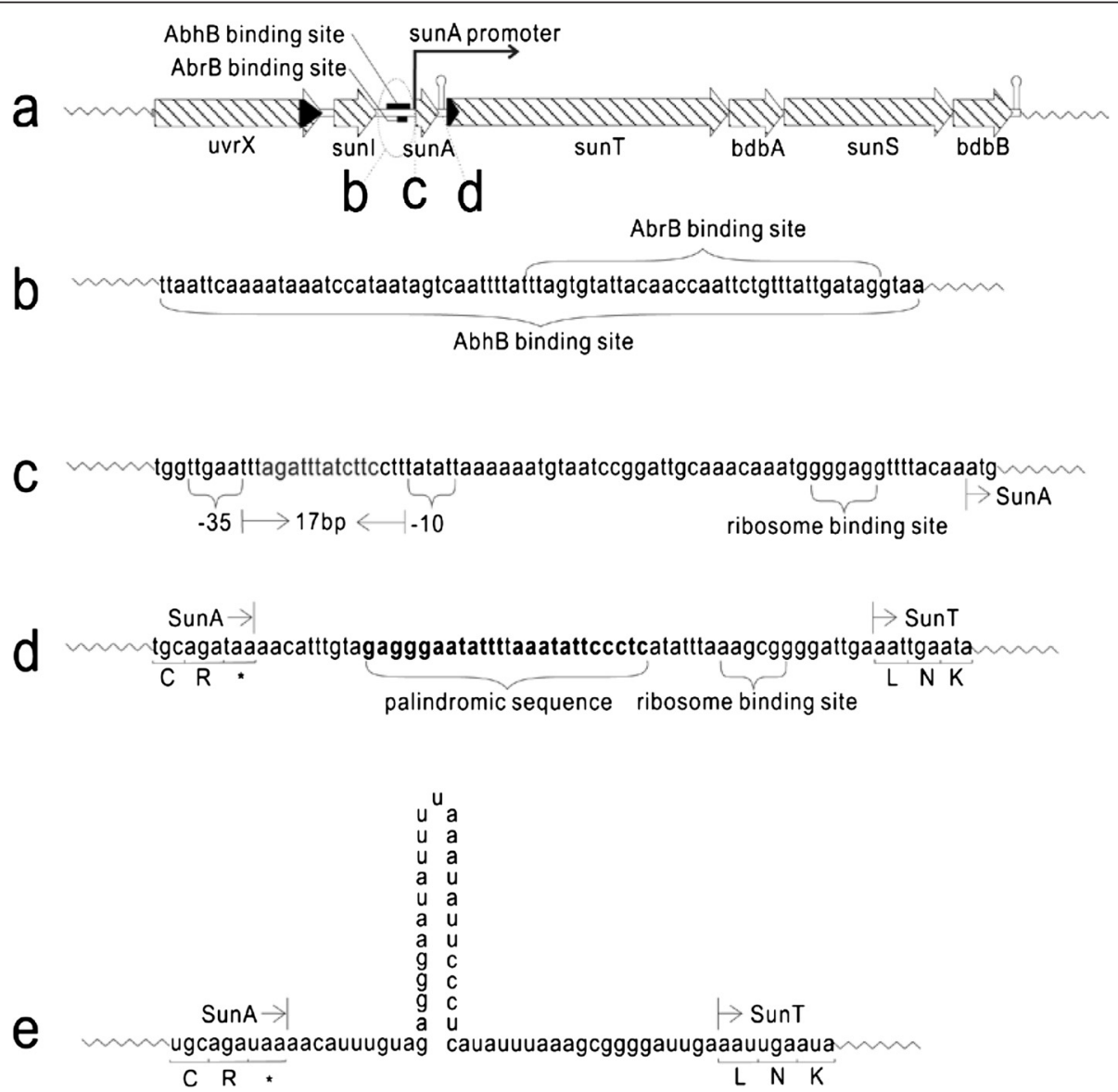

Figure 1 Schematic of the sublancin gene cluster. (a) The gene cluster of sublancin in B. subtilis 168 consists of the immunity protein gene sunl, precursor gene sunA, $A B C$-transporter gene sunT, two thiol-disulfide oxidoreductase genes $b d b A$ and $b d b B$, and glycosyltransferase gene sunS. (b) Abr and AbhB binding sites are located upstream of the promoter of sunA. (c) A typical $\sigma^{A}$ promoter is located upstream of sunA. (d) A palindromic sequence and a ribosome-binding site without promoter are located between sunA and sunT. (e) A hairpin structure is formed from the palindromic sequence after transcription. 
mediated by these factors. B. subtilis harbors a minimum of seven known ECF $\sigma$ factors viz., $\sigma^{\mathrm{M}}, \sigma^{\mathrm{W}}, \sigma^{\mathrm{X}}, \sigma^{\mathrm{Y}}, \sigma^{\mathrm{Z}}, \sigma^{\mathrm{V}}$, and $\sigma^{\mathrm{YlaC}}[18]$. Of them, two ECF $\sigma$ factors $\sigma^{\mathrm{M}}$ and $\sigma^{\mathrm{X}}$ with overlapping promoter specificity are involved in the biosynthesis-regulating of sublancin in B. subtilis 168 cells (Additional file 1) [19]. The monocistron sunA-sunT$b d b A-\operatorname{sun} S-b d b B$ with a hairpin structure in between $\operatorname{sun} A$ and $\operatorname{sun} T$ (Figure $1 \mathrm{~d}$ and e) is transcriptionally controlled under a $\sigma^{\mathrm{A}}$ promoter (Figurela and c), and its typical motifs of -35 and -10 region are TTGACA and TATAAT with a consensus spacing of 17 nucleotides.

Natural production of sublancin biosynthesized by $B$. subtilis 168 is poor owing to its complex transcriptional regulatory mechanism [2]. Comparing to single polypeptide consisting of common amino acids [20], the mature sublancin undergoes further post-translational modification, including formation of the characteristic glucosylation moiety and disulfide bridges. It is not suitable for commercial production through conventional recombinant DNA technology or common peptide chemosynthesis method $[1,2,5]$. Considering the possibility of displacing the complex transcriptional regulatory mechanism to efficiently biosynthesize sublancin, we altered the transcriptional regulatory network of $\operatorname{sunA}$ in situ with a strong inducible $\mathrm{P}_{g l v}$ vegetative $\sigma^{\mathrm{A}}$ promoter [21]. Meanwhile, other two strong promoters of P43 [22] and $\mathrm{P}_{\text {luxS }}$ [23] were placed before sunI and $\operatorname{sun} T-b d b A-s u n S-b d b B$, respectively.

\section{Results and discussion}

\section{Construction of recombinant $B$. subtilis 1 A747 strain}

Three strong characteristic promoters including vegetative-and-stationary double functional promoter $\mathrm{P} 43$ [22], the maltose-inducible promoter $\mathrm{P}_{g l v}[21]$ and vegetative promoter $\mathrm{P}_{\text {luxs }}$ [23], were in situ chromosome-integrated into B. subtilis $1 \mathrm{~A} 747$ and respectively placed before two genes of $\operatorname{sunI}$ and $\operatorname{sun} A$ and one gene cluster of sun $T-b d b A-s u n S-b d b B$ that are responsible for mature sublancin biosynthesis. Then, recombinant $B$. subtilis $1 \mathrm{~A} 747$ strains were constructed for the efficient biosynthesis of sublancin for commercial applications (Figure 2).

\section{Contribution of $\mathrm{P} 43, \mathrm{P}_{g / v}$, and $\mathrm{P}_{\text {luxs }}$ on over-transcription of relative genes}

Recombinant B. subtilis $1 \mathrm{~A} 747$ strains were cultured in modified Medium A $[2,24]$ at $37^{\circ} \mathrm{C}$ and induced at $12^{\text {th }}$ hour after fermentation start according to bacterial strain growth curve (Figure 3a). Meantime, the same culture system without maltose treatment served as a positive control and non-recombinant B. subtilis $1 \mathrm{~A} 747$ strain with maltose treatment served as a negative control (Figure 3a). That the transcript amount of $\operatorname{sun} A$ and relative genes in recombinant strain induced by $5 \%$ maltose were 2 to 5 times higher than those of negative control when these strains reached the late logarithmic phase (Figure 3a and b) suggested that recombinant B. subtilis 1A747 strains have an obvious advantage in effective biosynthesis of mature sublancin (Figure 3c).

\section{Improved biosynthesis of mature sublancin}

A maximum amount of $642 \mathrm{mg}$ sublancin from $1 \mathrm{~L}$ culture supernatant of recombinant strain was achieved (Figure 3c), which is statistically significantly higher than those of $67 \mathrm{mg} / \mathrm{L}$ with maltose treatment (Figure 3c) and at most $60 \mathrm{mg} / \mathrm{L}$ without maltose treatment [2] both obtained from non-recombinant parental B. subtilis 1 A747. Electrospray ionization mass spectrometry analysis showed that molecular weight of purified mature sublancin was 3877.642 KDa (Additional file 2), which is consistent with previous reports $[2,5]$ and the result of tricine-SDS-PAGE analysis of $3.9 \mathrm{KDa}$ (Figure 3d-a). Western blot analysis confirmed the result of tricine-SDS-PAGE analysis and showed that sublancin existed in culture supernatant (Figure 3d-b). The analysis of circular dichroism spectrum showed that purified mature sublancin in the liposome solution had a double-negative peak at 207 and $222 \mathrm{~nm}$ and thus displayed an $\alpha$-helical conformation (Additional file 3 ) that is also in agreement with other study [25].

The above findings suggest the importance and advantages of this recombinant DNA technology in effective and efficient biosynthesis of mature sublancin. Recently, the synthesis of this type $\mathrm{S}$-linked glycopeptide has become an interesting target through a complicated chemical [26] or semi-chemical [5] method, and several Slinked glycopeptides have been chemically synthesized [27-30]. However, both chemical and semi-chemical methods are suitable for small and linear glycopeptide synthesis but not for complicated glycopeptides like sublancin. The biosynthesis of S-linked glycopeptides with disulfide bridges such as sublancin using recombinant DNA technology has been rarely reported.

Several gene clusters such as those responsible for synthesizing mature nisin or subtilin in other microorganisms [31] are similar to that of sublancin, and also include the genes for precursor peptides and post-transcriptional modification enzymes. Their core peptides have different sequence identities but also contain abundant cysteine residues. In future, these specific post-transcriptionally modified antimicrobial peptides may become more common than currently appreciated. Therefore, it is assumed that more antimicrobial agents with post-transcriptional modification such as S-linked glycopeptides could be efficiently biosynthesized when to increase the possibilities of in situ utilizing vegetative or other appropriate promoters to adjust the transcriptional regulatory circuits of relative gene clusters. 


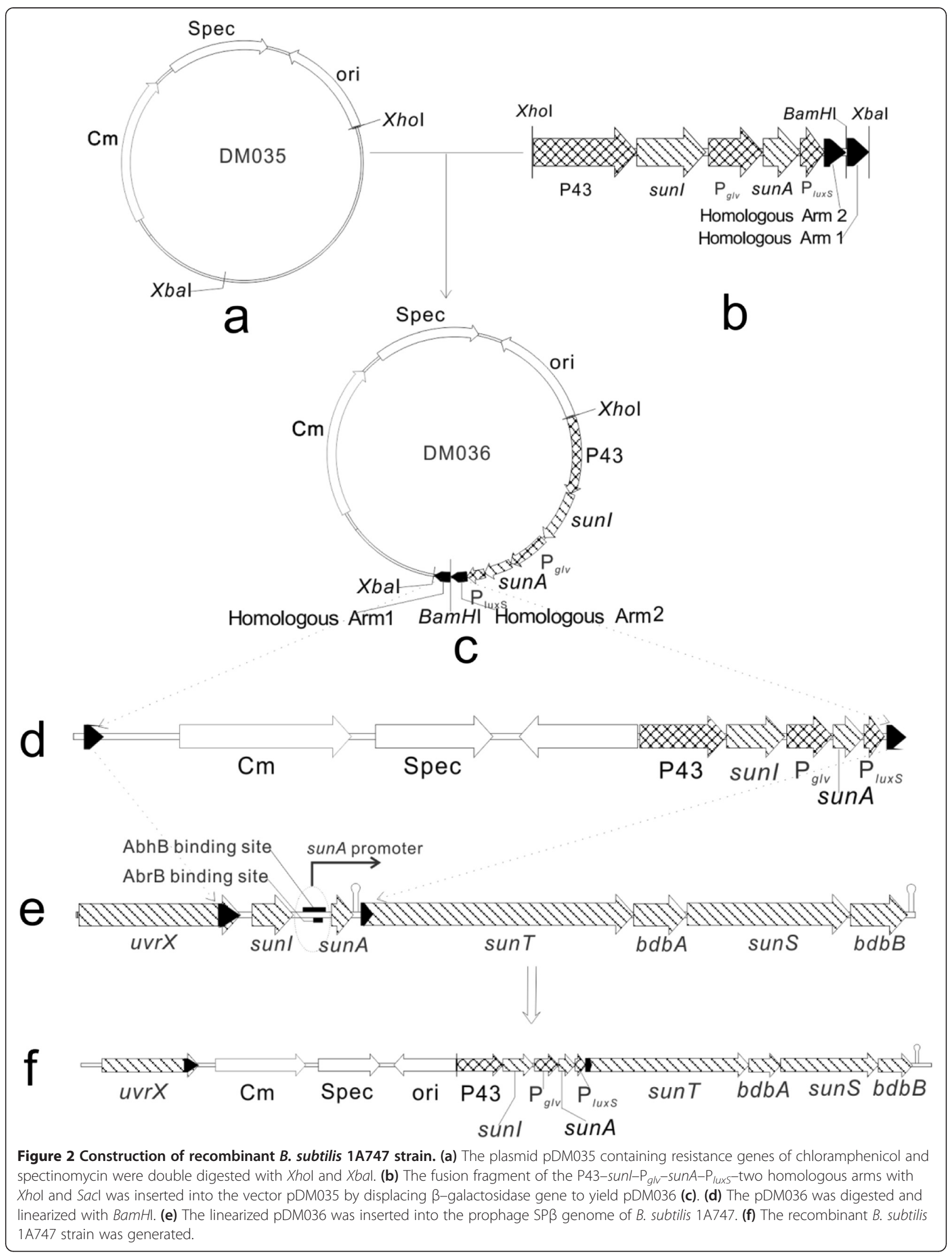




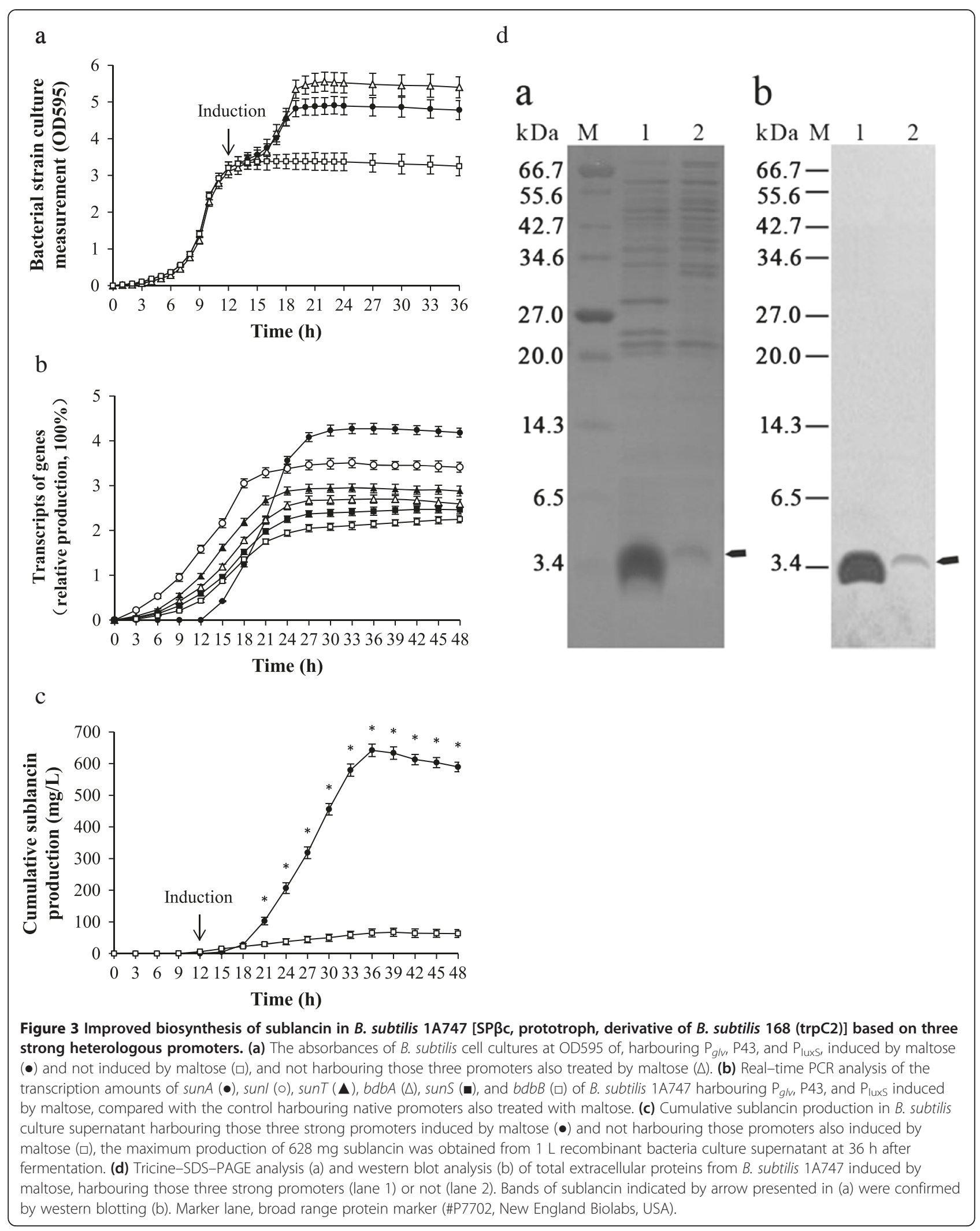




\section{Determination of conditional stability}

As a potential alternative to conventional antibiotics in treating some bacterial-mediated inflammations like mastitis and gastroenteritis, the conditional stabilities of sublancin were evaluated using the simulated in vivo conditions i.e., specific $\mathrm{pH}$, different temperatures and protease-rich environments. Our data show that temperatures ranging from $20^{\circ} \mathrm{C}$ to $70^{\circ} \mathrm{C}$ for 30 min can slightly affect the antimicrobial potency of sublancin (Figure 4a), while $\mathrm{pH}$ from 4.0 to 9.0 did not show significant effect on the antimicrobial activity (Figure 4b). The $\mathrm{pH}$ in body blood maintains at between 7.25 to 7.45 [32], whereas gastric juice, proximal small intestine, terminal ileum and cecum constantly maintain mean $\mathrm{pH}$ at 1.0 to $2.5,6.6,7.5$, and 6.4 , respectively [33]. Thus, these tissues besides stomach could provide optimum environments for potent biological activities of sublancin.

Proteolytic susceptibility of sublancin should also be considered in various applications such as in treating bacterial-mediated mastitis or bacterial-mediated gastroenteritis. Therefore, a number of bacterial proteases and gastrointestinal digestive enzymes, e.g., $P$. aeruginosa elastase and $S$. aureus V8 protease, pepsin and trypsin would certainly be encountered [34]. The results showed that sublancin maintained a majority of its activities when exposed to these proteases (Figure 4c). All experiments were performed in vitro; therefore in vivo studies should be conducted prior to clinical applications. Similar study about conditional stability of sublancin has not been previously documented. In contrast, a remarkable antimicrobial potency reduction of Bovicin HC5 was observed in Streptococcus bovis HC5 when exposed with proteinases, peptidases and heat [35], which can represent a common problem with regard to easy degradability of antimicrobial peptide.

Sugar linkages like glucose to cysteine can produce more stable products than conjugation to serine at high or low $\mathrm{pH}$ [36-38]. Mechanism of conditional stability of sublancin may be attributed to two disulfide bridges. Their coupling with glucose glycosylation probably provide the exceptional stability for sublancin by reducing the configurational entropy of unfolding; thus, conformational constraints are exerted, and conformational and biochemical stability are conferred on this agent [5].

\section{Activity of sublancin}

Antibacterial activity and cytotoxicity of sublancin were also evaluated. Sublancin showed a potent antimicrobial activity towards nine bacterial strains including five typical and four drug-resistant strains, especially against $S$. aureus with $\mathrm{MIC}<0.4 \mathrm{mg} / \mathrm{L}$ (Table 1 ). These findings are consistent with previously reported study [2] and is almost equal to that of nisin that is a common and typical antimicrobial peptide from Lactococcus lactis, and is widely used in

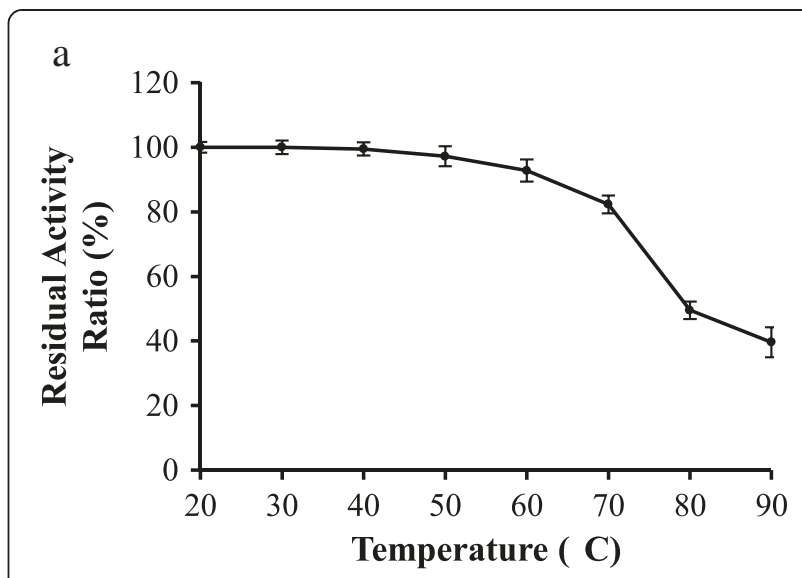

$\mathrm{b}$

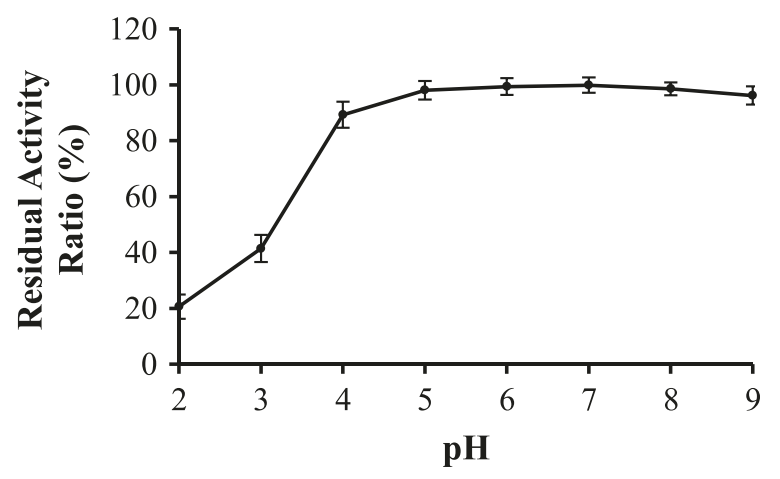

c

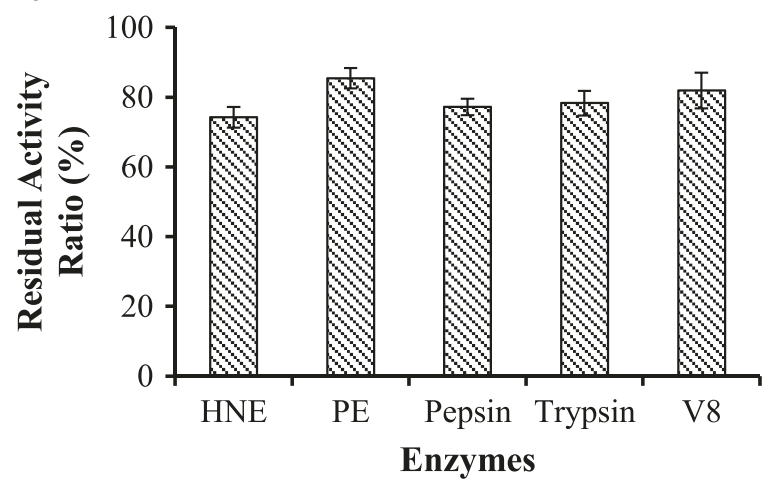

Figure 4 Effects of different temperatures (a), $\mathrm{pH}(\mathrm{b})$, and enzymes (c) on sublancin. The original reaction systems treated at $30^{\circ} \mathrm{C}$ and $\mathrm{pH} 7.0$ were designated as the positive control, whereas the identical reaction systems without sublancin were used correspondingly as the negative controls. The residual activity ratios were calculated according to the description in the text.

clinical and food preservation applications [39], with MIC of $0.5 \mathrm{mg} / \mathrm{L}$ against $S$. aureus. In addition, sublancin exhibited an $\mathrm{IC}_{50}>200 \mathrm{mg} / \mathrm{L}$ toward HT-29 cells, indicating its moderate cytotoxicity.

An almost unaffected growth curve of recombinant $B$. subtilis 1A747 strain under high sublancin concentration was observed after the maltose induction (Figure 3a and c), hence confirming that the producer possessed the 
Table 1 MICs of sublancin for different strains

\begin{tabular}{ll}
\hline Strains & MIC (mg/L) \\
\hline E. faecalis ATCC 29212 & $7.3 \pm 0.35$ \\
gentamicin-resistant E. faecalis & $6.8 \pm 0.29$ \\
S. aureus ATCC 25923 & $0.6 \pm 0.14$ \\
methicillin-resistant S. aureus & $0.4 \pm 0.21$ \\
S. agalactiae ATCC 27956 & $2.1 \pm 0.35$ \\
erythromycin-resistant S. agalactiae & $1.4 \pm 0.24$ \\
S. pyogenes ATCC 19615 & $0.8 \pm 0.22$ \\
erythromycin-resistant S. pyogenes & $1.0 \pm 0.36$ \\
B. cereus ATCC 10987 & $3.4 \pm 0.21$ \\
\hline
\end{tabular}

The data derived from average values for three independent replicate experiments and almost identical triplicate sets of data.

immunity against sublancin owning to the contribution of SunI generated simultaneously with sublancin [6].

\section{Conclusions}

In our present study, we for the first time developed a recombinant $B$. subtilis 1 A747 strain by displacing native promoters of genes responsible for mature sublancin biosynthesis with three distinctive promoters of $\mathrm{P} 43, \mathrm{P}_{g l v}$ and $\mathrm{P}_{l u x S}$, and the developed recombinant strain is capable of efficiently producing sublancin. The biosynthesized mature sublancin showed a rational molecular weight and conformation, displayed a potent and broad activity spectrum with a moderate cytotoxicity and illustrated a good conditional stability under the treatment with high temperatures, extreme $\mathrm{pH}$ and specific proteases. These findings are important for this recombinant strain in allowing for effective and efficient biosynthesis of sublancin. The promoter replacement provides an extra choice for high production of some other complicated peptide such as nisin and subtilin.

\section{Materials and methods}

\section{Construction of mutant strains of $B$. subtilis 168}

The pDM035 (Figure 2a) (kept in our laboratory), a shuttle vector able to replicate both in E.coli and B. subtilis and containing resistance genes against chloramphenicol and spectinomycin, was double digested with $X h o \mathrm{I}$ and $X b a \mathrm{I}$. The fusion fragment of $\mathrm{P} 43-$ sunI- $\mathrm{P}_{g l v}-$ sunA-P $\mathrm{P}_{l u x S}$-two homologous arms with XhoI and $S a c I$ (Figure 2b) was synthesized at AuGCT Co. Ltd. (Beijing, China). The fragment was inserted into vector pDM035 by displacing $\beta$-galactosidase gene to generate pDM036 (Figure 2c). The resultant pDM036 was transferred into Escherichia coli, and the positive clones were selected using $5 \mu \mathrm{g} / \mathrm{mL}$ chloramphenicol. B. subtilis 1A747 [SP $\beta$ c, prototroph, derivative of B. subtilis 168 (trpC2)] (Bacillus Genetic Stock Center, USA) competent cells were transformed with linearized pDM036 digested with BamHI using the electroporation approach [40].
The positive-recombinant $B$. subtilis cells were selected from LB agar with $5 \mu \mathrm{g} / \mathrm{mL}$ chloramphenicol and $50 \mu \mathrm{g} / \mathrm{mL}$ spectinomycin resistances. These strains were cultured in $50 \mathrm{~mL}$ modified Medium A [2,24] in $250 \mathrm{~mL}$ shake flask with agitation speed of $225 \mathrm{rpm}$ at $37^{\circ} \mathrm{C}$. The culture mixture was not supplied with $5 \%$ maltose until the late logarithmic phase of strains [41]. After another $24 \mathrm{~h}$ culture, the fermentation broth was harvested and centrifuged at $12,000 \times g$ for $15 \mathrm{~min}$ at $4^{\circ} \mathrm{C}$ and the supernatants were collected. B. subtilis $1 \mathrm{~A} 747$ strains were used as control that was performed as recombinant $B$. subtilis was done. Crude sublancin concentrations in fermentation supernatant were measured by HPLC using the method described below.

\section{Isolation of total RNA and Real-time PCR}

The cultures were collected once every $3 \mathrm{~h}$ from $3 \mathrm{~h}$ until $48 \mathrm{~h}$ after fermentation. Total bacterial RNAs were isolated by a SV total RNA isolation kit (\#Z3100; Promega, USA). Extracted total RNA was reverse-transcribed into cDNA chain by a Reverse Transcription System Kit (\#A3500; Promega, USA). Real-time PCR was carried out using Real time PCR Kit (\#DRR041S; TaKaRa, Japan). The genes of sunI, sunA, sunT, $b d b A$, sunS and $b d b B$ were amplified by primers as shown in Table 2. $16 \mathrm{~s}$ rDNA of $B$. subtilis 168 was amplified as control using $16 \mathrm{~s}-\mathrm{up} / 16 \mathrm{~s}-$ down primers. PCR protocols were as follows: $2 \mathrm{~min}$ at $50^{\circ} \mathrm{C}$ and $10 \mathrm{~min}$ at $95^{\circ} \mathrm{C}$, followed by 35 cycles consisting of $42 \mathrm{~s}$ at $95^{\circ} \mathrm{C}, 60 \mathrm{~s}$ at the annealing temperatures shown in Table 2, and $30 \mathrm{~s}$ at $72^{\circ} \mathrm{C}$. Reactions were performed in IQ5 Real-time PCR detection system (Bio-RAD, USA).

\section{Western blotting}

The culture supernatants at $36 \mathrm{~h}$ were collected at $12000 \mathrm{~g}$ for $10 \mathrm{~min}$, mixed with $4 \times$ Laemmli loading buffer (3:1) and heated in boiling water for $5 \mathrm{~min}$. Subsequently, the tricine sodium dodecyl sulfate polyacrylamide gel electrophoresis (tricine-SDS-PAGE) [42] analysis was performed using $10 \%$ gel (Figure $3 \mathrm{~d}-\mathrm{a}$ ) and then electro-transferred to PVDF membrane (Millipore, USA) for protein immunoblot analysis (Figure $3 \mathrm{~d}-\mathrm{b}$ ). The preparation and purification of mouse anti-sublancin monoclonal antibody was performed by Cwbiotech (Beijing, China). After incubation with HRPconjugate secondary antibody, bands were visualized by chemiluminescence using a ChemiDoc XRS imaging system and analysis software Quantity One (Bio-Rad, USA).

\section{Purification of sublancin}

Isolation and purification of sublancin were performed as described previously [2] with slight modification. The harvested supernatant was placed in $1 \mathrm{M} \mathrm{NaCl}$ and then subjected to a hydrophobic interaction chromatography using $25 \mathrm{~mL}$ Toyo pearl Butyl-650 column (Tosoh, Tokyo, Japan), equilibrated with $1 \mathrm{M} \mathrm{NaCl}$ and $50 \mathrm{mM}$ 
Table 2 Primers and annealing temperatures used in real-time PCR

\begin{tabular}{|c|c|c|c|}
\hline Gene & Primer & Sequence & Annealing temperature $\left({ }^{\circ} \mathrm{C}\right)$ \\
\hline \multirow[t]{2}{*}{ sunl } & sunl-up & AAGAGTCAGACAAGTATGGAGTT & 48 \\
\hline & sunl-down & TTAAATGGAGCTCAACAATTTA & \\
\hline \multirow[t]{2}{*}{$\operatorname{sun} A$} & sunA-up & GAACTGGAAAATCAAAAAGGT & 49 \\
\hline & sunA-down & CAAAACTGCCGGTAATTCT & \\
\hline \multirow[t]{2}{*}{ sunt } & sunT-up & GGGGATAAGGAAGGCTATAG & 50 \\
\hline & sunT-down & TAATGTCCATATTCCTCCCC & \\
\hline \multirow[t]{2}{*}{$b d b A$} & bdbA-up & GCAGCAGCCATTAGTATTTTC & 51 \\
\hline & bdbA-down & CAAGGAGGACAACTTGTCTCA & \\
\hline \multirow[t]{2}{*}{ suns } & sunS-up & GGCTATGCCGATTCTITATT & 50 \\
\hline & sunS-down & CCGCATGTTATTGTAGGAGTA & \\
\hline \multirow[t]{2}{*}{$b d b B$} & bdbB-up & CCATGTGTTCTATGTTGGTATC & 49 \\
\hline & bdbB-down & CCAATTTCACATACGACACTT & \\
\hline
\end{tabular}

$\mathrm{NaAc}(\mathrm{pH} 4.0)$. The unbound proteins were washed with loading buffer, and $50 \mathrm{mM} \mathrm{NaAc}(\mathrm{pH} 4.0)$ was used to elute sublancin. The elution was placed in $0.1 \%$ TFA and subjected to HPLC using a semi-preparative Zorbax 300SB-C8 column $(250 \mathrm{~mm} \times 9.4 \mathrm{~mm}, 5 \mu \mathrm{m}$ particle size, $300 \AA$ pore size; Agilent, Englewood, CO), equilibrated in $0.1 \%(\mathrm{v} / \mathrm{v})$ TFA and $10 \%$ acetonitrile. The elution was subsequently developed with a linear $0 \%$ to $60 \%$ acetonitrile gradient at a flow rate of $1.0 \mathrm{~mL} / \mathrm{min}$. Fractions from different retention times were tested for antimicrobial activity. Active fractions were collected and then subjected to HPLC using an analytical Zorbax 300SB-C8 column $(150 \mathrm{~mm} \times 4.6 \mathrm{~mm}, 5 \mu \mathrm{m}$ particle size, $300 \AA$ pore size; Agilent, Englewood, CO) under the same conditions as used in the first step. The absorbances at 214, 254 and $280 \mathrm{~nm}$ were monitored and $S$. aureus was designated as indicator strain in antimicrobial activity assays. The concentration of purified sublancin was determined by UV spectrophotometry [43,44]. The molecular weights of sublancin were obtained by electrospray ionization mass spectrometry (Agilent, USA; Figure 4). The pooled solution of sublancin was freeze-dried in a vacuum freeze dryer (SIM International Group Co., Ltd., USA) at $-80^{\circ} \mathrm{C}$ for further experiment.

\section{Secondary structure analysis}

Using a $1 \mathrm{~mm}$ path-length quartz cuvette, the second structure of sublancin at $50 \mathrm{mg} / \mathrm{L}$ in Palmitoyl-oleoyl-phosphatidylglycerol (POPG) liposome solution was detected using a Jasco 810 spectropolarimeter (Jasco Corporation, Japan) at room temperature, within the range of $190 \mathrm{~nm}$ to $250 \mathrm{~nm}$ at $10 \mathrm{~nm} / \mathrm{min}$, as described previously [45]. The liposome solution without sublancin was used as a reference. POPG liposomes (Sigma, USA) were prepared with the following slight modification: a specific amount of POPG was dissolved into $10 \mathrm{mM}$ PBS (pH 7.4) to prepare the stock solution of $100 \mu \mathrm{M}$ POPG.

\section{Antimicrobial activity}

B. cereus ATCC 10987, Enterococcus faecalis ATCC 29212, S. aureus ATCC 25923, S. agalactiae ATCC 27956, and $S$. pyogenes ATCC19615 were obtained from ATCC (Rockville, MD, USA). Erythromycin-resistant S. agalactiae, erythromycin-resistant $S$. pyogenes, gentamicin-resistant $E$. faecalis and methicillin-resistant $S$. aureus were kept in our laboratory. MIC assay was performed depending on a microtiter broth dilution method, as described previously [46], with slight modification. A packed volume with DEAE-Sephacel (Sigma-Aldrich, Schnelldorf, Germany) was equilibrated with deionized water, $2 \mathrm{M} \mathrm{NaCl}, 0.1 \mathrm{M}$ $\mathrm{NaOH}, 70 \%$ ethanol, and $10 \mathrm{mM}$ Tris buffer ( $\mathrm{pH}$ 7.4). Approximately $100 \mathrm{~mL}$ of $\mathrm{LB}$ broth in the same Tris buffer was subjected to the treated column to prepare a refined medium, and then sterilized using $0.22 \mu \mathrm{L}$ of the membrane filter. In addition, the peptide sample was dissolved into Tris buffer to prepare 10-fold serial dilutions. After overnight culture, the tested strains were rinsed with Tris buffer and diluted to $5 \times 10^{5} \mathrm{CFU} / \mathrm{mL}$ in refined $\mathrm{LB}$ medium. Aliquots of $90 \mu \mathrm{L}$ of bacteria solution were added into the wells of a 96-well microtiter plate. Subsequently, $10 \mu \mathrm{L}$ of the serial $10-$ fold dilutions were placed into corresponding wells and produced a serial working concentration of $64,32,16,8,4,2,1,0.5,0.25,0.12$ and $0.06 \mathrm{mg} / \mathrm{L}$. The mixtures were incubated at $37^{\circ} \mathrm{C}$ for $21 \mathrm{~h}$, and then measured as described previously [47]. A negative control was also prepared using same reaction system without sublancin. These experiments were repeated three times (hereinafter the same). MIC was defined as the lowest concentration of an antimicrobial agent required to inhibit 90\% of microorganism growth after overnight incubation. 


\section{HT-29 lytic activity}

The human colorectal adenocarcinoma cell line HT-29 were obtained from the ATCC and cultured in RPMI-1640 medium (Invitrogen, USA) supplemented with $0.2 \mathrm{~g} / \mathrm{L}$ streptomycin, $0.1 \mathrm{~g} / \mathrm{L}$ penicillin, $10 \%$ heat-inactivated fetal calf serum (FCS, Germany). Cells were maintained at $37^{\circ} \mathrm{C}$ in $5 \% \mathrm{CO}_{2}$. For assays, the HT-29 cells were first starved for $24 \mathrm{~h}$ in serum-free medium, and then were seeded in a 24-well plate (Nunc, Germany) at $1 \times 10^{5}$ cells/well. At subconfluency, medium was replaced, and the cells were incubated with the serial sublancin dilutions of 200, 100, 50, $25,12,6$, and $3 \mathrm{mg} / \mathrm{L}$ in a volume of $100 \mu \mathrm{l}$ for $24 \mathrm{~h}$. Cell viability was assessed in exposed cultures by using a colorimetric 3-(4,5-dimethylthiazol-2-yl)-2,5 diphenyltetrazoliumthiazolyl blue assay (MTT, Roche Diagnostics, Germany). The reaction samples were detected at $570 \mathrm{~nm}$ with a microtiter ELISA reader $\left(\right.$ Epoch $^{\mathrm{TM}}$, BioTek- ${ }^{\bullet}$ instruments, Inc., USA).

\section{Assessment of stability}

The effect of different factors including enzymes, $\mathrm{pH}$ and temperatures on sublancin stability was evaluated. The evaluation was performed in accordance to the method described in the antimicrobial activity section with slight modification. Aliquots of $5 \mathrm{mg} / \mathrm{L}$ sublancin in PBS ( $\mathrm{pH}$ 7.4) were treated under different protease [HNE (Innovative Research, Novi, MI), PE (Innovative Research), pepsin (Sigma), V8 (BioCol GmbH), and trypsin (Sigma Chemical Co., St. Louis, MO)] at a substrate: protease molar ratio of $300: 1$ at $37^{\circ} \mathrm{C}$, gradient $\mathrm{pH}$ values $(\mathrm{pH} 2.0,3.0,4.0,5.0,6.0,7.0,8.0$, and 9.0$)$ at $37^{\circ} \mathrm{C}$ and gradient temperatures $\left(20,30,40,50,60,70,80\right.$ and $\left.90^{\circ} \mathrm{C}\right)$, all treatments were performed for $30 \mathrm{~min}$. After the treatment, the indicator bacteria were mixed with $100 \mu \mathrm{L}$ treated peptide solution. Approximately $5 \times 10^{5} \mathrm{CFU} / \mathrm{mL}$ solution was obtained and then incubated at $37^{\circ} \mathrm{C}$ for $21 \mathrm{~h}$. At the same time, a similar reaction system at $30^{\circ} \mathrm{C}$ (pH 7.0) was set as positive control; the same systems without sublancin were correspondingly used as negative controls. S. aureus was used as indicator strain. The effect of these factors on sublancin stability was evaluated through residual activity using the following formula:

$$
\text { residual activity ratio }(\%)=\left(A_{1}-A\right) /\left(A_{1}-A_{2}\right) \times 100 \%
$$

where $A, A_{1}$, and $A_{2}$ represent the absorbance of the different factors, negative controls and positive control, respectively.

\section{Statistical analysis}

The experiments were repeated for three times, and the mean values were expressed as mean \pm standard deviation.

\section{Additional files}

Additional file 1. Regulation net of sublancin biosynthesis.

Additional file 2. Electrospray ionization mass spectrometry analysis for mature sublancin.

Additional file 3. Circular dichroism spectrum of sublancin in liposome solution.

\section{Abbreviations}

ECF $\sigma$ factors: Extra cytoplasmic function $\sigma$ factors; LB: Lysogeny broth; HPLC: High performance liquid chromatography; MTT: 3-(4, 5-dimethylthiazol-2-yl)-2, 5 diphenyltetrazoliumthiazolyl blue; TFA: Trifluoroacetic acid; POPG:

Palmitoyl-oleoyl-phosphatidylglycerol; CFU: Colony-forming units; ATCC: American Type Culture Collection; MIC: Minimum inhibitory concentration; HNE: Human neutrophil elastase; PEY: P. aeruginosa elastase; V8:S. aureus: V8 protease.

\section{Competing interests}

The authors declare that they have no competing interests.

\section{Authors' contributions}

Binyun Cao and Shengyue Ji designed and carried out experiments, analyzed data and helped to write the manuscript. Weili Li designed experiments, analyzed data and helped to write the manuscript. Rasheed and Meng Wang designed and carried out experiments and analyzed data. All authors read and approved the final manuscript.

\section{Acknowledgements}

This work was supported by the National Science and Technology R\&D Program of China (Grant No. 2011BAD28B05-3) and the Provincial Agriculture Special Fund Project of China (Grant No. 2011NYTT03). We wish to thank Dr Mingming Yang for his assistance on the construction of the recombinant $B$. subtilis 1 A747strains.

\section{Author details}

${ }^{1}$ College of Animal Science and Technology, Northwest A\&F University, 22 Xinong Road, Yangling, 712100 Shaanxi, P.R. China. ${ }^{2}$ College of Veterinary Medicine, Northwest A\&F University, 22 Xinong Road, Yangling, 712100 Shaanxi, P.R. China.

Received: 14 October 2014 Accepted: 26 January 2015

Published online: 12 February 2015

\section{References}

1. Oman TJ, van der Donk WA. Follow the leader: the use of leader peptides to guide natural product biosynthesis. Nat Chem Biol. 2010;6:9-18.

2. Paik SH, Chakicherla A, Hansen JN. Identification and characterization of the structural and transporter genes for, and the chemical and biological properties of, sublancin 168, a novel lantibiotic produced by Bacillus subtilis 168. J Biol Chem. 1998;273:23134-42.

3. Hemphill HE, Gage I, Zahler SA, Korman RZ. Prophage-mediated production of a bacteriocinlike substance by SP beta lysogens of Bacillus subtilis. Can J Microbiol. 1980;26:1328-33.

4. Willey JM, van der Donk WA. Lantibiotics: peptides of diverse structure and function. Annu Rev Microbiol. 2007;61:477-501.

5. Oman TJ, Boettcher JM, Wang H, Okalibe XN, van der Donk WA. Sublancin is not a lantibiotic but an S-linked glycopeptide. Nat Chem Biol. 2011;7:78-80.

6. Dubois JY, Kouwen TR, Schurich AK, Reis CR, Ensing HT, Trip EN, et al. Immunity to the bacteriocin sublancin 168 Is determined by the Sunl (YolF) protein of Bacillus subtilis. Antimicrob Agents Chemother. 2009;53:651-61.

7. Serizawa M, Kodama K, Yamamoto H, Kobayashi K, Ogasawara N, Sekiguchi J. Functional analysis of the YvrGHb two-component system of Bacillus subtilis: identification of the regulated genes by DNA microarray and northern blot analyses. Biosci Biotechnol Biochem. 2005;69:2155-69.

8. Nicolas P, Mader U, Dervyn E, Rochat T, Leduc A, Pigeonneau N, et al. Condition-dependent transcriptome reveals high-level regulatory architecture in Bacillus subtilis. Science. 2012;335:1103-6. 
9. Dorenbos R, Stein T, Kabel J, Bruand C, Bolhuis A, Bron S, et al. Thiol-disulfide oxidoreductases are essential for the production of the lantibiotic sublancin 168. J Biol Chem. 2002;277:16682-8.

10. McAuliffe O, Ross RP, Hill C. Lantibiotics: structure, biosynthesis and mode of action. FEMS Microbiol Rev. 2001;25:285-308.

11. Kouwen TR, van der Goot A, Dorenbos R, Winter T, Antelmann H, Plaisier MC, et al. Thiol-disulphide oxidoreductase modules in the low-GC Gram-positive bacteria. Mol Microbiol. 2007:64:984-99.

12. Ritz D, Beckwith J. Roles of thiol-redox pathways in bacteria. Annu Rev Microbiol. 2001:55:21-48.

13. Wang $H$, van der Donk WA. Substrate selectivity of the sublancin S-glycosyltransferase. J Am Chem Soc. 2011;133:16394-7.

14. Strauch MA, Bobay BG, Cavanagh J, Yao F, Wilson A, Le Breton Y. Abh and AbrB control of Bacillus subtilis antimicrobial gene expression. J Bacteriol. 2007;189:7720-32

15. Albano M, Smits WK, Ho LT, Kraigher B, Mandic-Mulec I, Kuipers OP, et al. The Rok protein of Bacillus subtilis represses genes for cell surface and extracellular functions. J Bacteriol. 2005;187:2010-9.

16. Chumsakul O, Takahashi H, Oshima T, Hishimoto T, Kanaya S, Ogasawara N et al. Genome-wide binding profiles of the Bacillus subtilis transition state regulator $\mathrm{AbrB}$ and its homolog $\mathrm{Abh}$ reveals their interactive role in transcriptional regulation. Nucleic Acids Res. 2011;39:414-28.

17. Missiakas D, Raina S. The extracytoplasmic function sigma factors: role and regulation. Mol Microbiol. 1998;28:1059-66.

18. Helmann JD. The extracytoplasmic function (ECF) sigma factors. Adv Microb Physiol. 2002:46:47-110.

19. Luo Y, Helmann JD. Extracytoplasmic function sigma factors with overlapping promoter specificity regulate sublancin production in Bacillus subtilis. J Bacteriol. 2009;191:4951-8.

20. Callaway JE, Lai J, Haselbeck B, Baltaian M, Bonnesen SP, Weickmann J, et al. Modification of the $C$ terminus of cecropin is essential for broad-spectrum antimicrobial activity. Antimicrob Agents Chemother. 1993:37:1614-9.

21. Ming YM, Wei ZW, Lin CY, Sheng GY. Development of a Bacillus subtilis expression system using the improved $\mathrm{P}_{g^{\prime}}$ promoter. Microb Cell Fact. 2010;9:55.

22. Zhang XZ, Cui ZL, Hong Q, Li SP. High-level expression and secretion of methyl parathion hydrolase in Bacillus subtilis WB800. Appl Environ Microbiol. 2005:71:4101-3.

23. Yang M, Zhang W, Ji S, Cao P, Chen Y, Zhao X. Generation of an artificial double promoter for protein expression in Bacillus subtilis through a promoter trap system. PLoS One. 2013;8:e56321.

24. Banerjee S, Hansen JN. Structure and expression of a gene encoding the precursor of subtilin, a small protein antibiotic. J Biol Chem. 1988;263:9508-14.

25. Garcia De Gonzalo CV, Zhu L, Oman TJ, van der Donk WA. NMR structure of the S-linked glycopeptide sublancin 168. ACS Chem Biol. 2014;9:796-801

26. Katayama $H$, Asahina $Y$, Hojo H. Chemical synthesis of the S-linked glycopeptide, sublancin. J Pept Sci. 2011;17:818-21.

27. Thayer DA, Yu HN, Galan MC, Wong CH. A general strategy toward S-linked glycopeptides. Angew Chem Int Ed Engl. 2005;44:4596-9.

28. Galonic DP, van der Donk WA, Gin DY. Site-selective conjugation of thiols with aziridine-2-carboxylic acid-containing peptides. J Am Chem Soc. 2004;126:12712-3

29. Galonic DP, Ide ND, van der Donk WA, Gin DY. Aziridine-2-carboxylic acid-containing peptides: application to solution- and solid-phase convergent site-selective peptide modification. J Am Chem Soc. 2005;127:7359-69.

30. Cohen SB, Halcomb RL. Application of serine- and threonine-derived cyclic sulfamidates for the preparation of S-linked glycosyl amino acids in solution- and solid-phase peptide synthesis. J Am Chem Soc. 2002;124:2534-43.

31. Smith $L$, Hillman J. Therapeutic potential of type A (I) lantibiotics, a group of cationic peptide antibiotics. Curr Opin Microbiol. 2008;11:401-8.

32. Rosenthal TB. The effect of temperature on the $\mathrm{pH}$ of blood and plasma in vitro. J Biol Chem. 1948;173:25-30.

33. Evans DF, Pye G, Bramley R, Clark AG, Dyson TJ, Hardcastle JD. Measurement of gastrointestinal $\mathrm{pH}$ profiles in normal ambulant human subjects. Gut. 1988;29:1035-41.

34. Low AG. The activity of pepsin, chymotrypsin and trypsin during $24 \mathrm{~h}$ periods in the small intestine of growing pigs. Br J Nutr. 1982;48:147-59.

35. Mantovani HC, Hu H, Worobo RW, Russell JB. Bovicin HC5, a bacteriocin from Streptococcus bovis HC5. Microbiology. 2002;148:3347-52.
36. Baran E, Drabarek S. Studies on synthesis of s-glycosidic bond between cysteine and glucose or galactose. Pol J Chem. 1978:52:941-6.

37. Jahn M, Marles J, Warren RA, Withers SG. Thioglycoligases: mutant glycosidases for thioglycoside synthesis. Angew Chem Int Ed Engl. 2003;42:352-4

38. Driguez $\mathrm{H}$. Thiooligosaccharides as tools for structural biology. Chembiochem. 2001;2:311-8.

39. Piper C, Draper LA, Cotter PD, Ross RP, Hill C. A comparison of the activities of lacticin 3147 and nisin against drug-resistant Staphylococcus aureus and Enterococcus species. J Antimicrob Chemother. 2009;64:546-51.

40. Xue G-P, Johnson JS, Dalrymple BP. High osmolarity improves the electro-transformation efficiency of the Gram-positive bacteria Bacillus subtilis and Bacillus licheniformis. J Microbiol Methods. 1999:34:183-91.

41. Ming-Ming Y, Wei-Wei Z, Xi-Feng Z, Pei-Lin C. Construction and characterization of a novel maltose inducible expression vector in Bacillus subtilis. Biotechnol Lett. 2006;28:1713-8.

42. Schagger H. Tricine-SDS-PAGE. Nat Protoc. 2006;1:16-22.

43. Edelhoch $\mathrm{H}$. Spectroscopic determination of tryptophan and tyrosine in proteins. Biochemistry. 1967;6:1948-54.

44. Gill SC, von Hippel PH. Calculation of protein extinction coefficients from amino acid sequence data. Anal Biochem. 1989;182:319-26.

45. Turner J, Cho Y, Dinh NN, Waring AJ, Lehrer Rl. Activities of LL-37, a cathelin-associated antimicrobial peptide of human neutrophils. Antimicrob Agents Chemother. 1998:42:2206-14.

46. Wu M, Hancock RE. Interaction of the cyclic antimicrobial cationic peptide bactenecin with the outer and cytoplasmic membrane. J Biol Chem. 1999;274:29-35.

47. Wiegand I, Hilpert $K$, Hancock RE. Agar and broth dilution methods to determine the minimal inhibitory concentration (MIC) of antimicrobial substances. Nat Protoc. 2008:3:163-75.

\section{Submit your next manuscript to BioMed Central and take full advantage of:}

- Convenient online submission

- Thorough peer review

- No space constraints or color figure charges

- Immediate publication on acceptance

- Inclusion in PubMed, CAS, Scopus and Google Scholar

- Research which is freely available for redistribution 